32 Lee YC, Kwak YG, Song CH. Contribution of vascular endothelial growth factor to airway hyperresponsiveness and inflammation in a murine model of toluene diisocyanate-induced asthma. $\mathrm{J}$ Immunol 2002; 168:3595-600.

33 Feltis BN, Wignarajah D, Reid DW, et al. Effects of inhaled fluticasone on angiogenesis and vascular endothelial growth factor in asthma. Thorax 2007;62:314-9.

34 Feltis BN, Wignarajah D, Zheng L, et al. Increased vascular endothelial growth factor and receptors: relationship to angiogenesis in asthma. Am J Respir Crit Care Med 2006;173:1201-7.

35 Volonaki E, Psarras S, Xepapadaki P, et al. Synergistic effects of fluticasone propionate and salmeterol on inhibiting rhinovirus-induced epithelial production of remodelling-associated growth factors. Clin Exp Allergy 2006;36:1268-73.

36 Yoo PS, Mulkeen AL, Cha CH. Post-transcriptional regulation of vascular endothelial growth factor:implications for tumor angiogenesis. World J Gastroenterol 2006; 12:4937-42.

37 Nie $M$, Knox AJ, Pang L. $\beta_{2}$-Ádrenoceptor agonists, like glucocorticoids, repress eotaxin gene transcription by selective inhibition of histone $\mathrm{H} 4$ acetylation. J Immunol 2005; 175:478-86.

38 Pang L, Knox AJ. Synergistic inhibition by beta(2)agonists and corticosteroids on tumor necrosis factor-alpha-induced interleukin-8 release from

\section{Bimodality surveillance of high-risk patients for lung cancer}

\section{Gordon H Downie}

\section{Are new diagnostic strategies providing answers?}

T horacic oncology providers confronted with the task of diagnosing and following patients at risk for cancer of the lung face a number of major dilemmas, some of which directly affect the ability to diagnose. First, the majority of patients with lung cancer are diagnosed at a late stage and $<15 \%$ survive 5 years, so a degree of nihilism is present in patients, providers and policy makers. Second, risk paradigms are changing, from smoking only to occupational, environmental or home carcinogens to the risk associated with premalignant airway changes. Third, advances in early diagnostic options have the potential to discover lung carcinoma while still in a pre-invasive, minimally invasive stage or as small peripheral nodules. These points, taken in conjunction with the initial clinical results of the ELCAP study suggesting that cure is possible, ${ }^{1}$ raise the need to examine early diagnostic strategies critically.

In this issue of Thorax (see page 335) Loewen et al report their initial clinical findings in bimodality surveillance of high risk for lung cancer populations using low dose spiral CT scanning (SCT) and autofluorescence bronchoscopy (AFB). ${ }^{2}$ They examined two null hypotheses: (1) AFB was equivalent to conventional sputum cytology (CSC) for the detection of pre-malignant lesions and (2) AFB and SCT would be equivalent to SCT alone for the detection of lung cancer in high-risk patients. The authors conclude that AFB is significantly superior to CSC for the detection of airway premalignancy in this cohort of high-risk patients and, in fact, argue that, as a surveillance tool, AFB exceeds the cancer detection rate of colonoscopy in patients with positive fecal occult blood. However, the authors were not able to demonstrate a significant superiority of bimodality surveillance with both AFB and SCT over SCT alone, but question whether a larger sample size would have found bimodality significantly better.

Beyond their null hypotheses, the article raises several points that are healthy components of any discussion of the future approach to patients at high risk of lung cancer. These include:

1. Premalignant changes are common (66\% of the 169 patients receiving all components of surveillance) in this high-risk cohort.

2. $\mathrm{AFB}$ is reasonable in patients with atypia in CSC; however, CSC was inadequate for detection of premalignant cytology when frank carcinoma was not present.

3. Screening and surveillance are very different and surveillance of a select population may be a superior strategy in lung cancer management.

4. Regardless of the histology of the lung cancers detected in this study ( $>50 \%$ were adenocarcinoma), the cultured human airway smooth-muscle cells. Am J Respir Cell Mol Biol 2000;23:79-85.

39 Kanazawa H, Yoshikawa T, Hirata K, et al. Effects of pranlukast administration on vascular endothelial growth factor levels in asthmatic patients. Chest 2004; 125:1700-5

40 Thompson HG, Truong DT, Griffith CK, et al. A three-dimensional in vitro model of angiogenesis in the airway mucosa. Pulm Pharmacol Ther 2007;20:141-8.

41 Tigani B, Cannet C, Quintana HK, et al. Lung inflammation and vascular remodeling after repeated allergen challenge detected noninvasively by MRI. Am J Physiol Lung Cell Mol Physiol, 2006 [Epub ahead of print]. majority of patients had central airway pre-malignant transformation.

5. Spiral CT scan protocols are not adequate at this time for detecting central airway disease by themselves.

6. Central airway pre-malignant lesions appear to be predictive of the presence of peripheral adenocarcinoma identified by SCT.

Several of these observations or conclusions have not been supported by other articles in the field. Haubinger et $a l^{3}$ performed a prospective, randomised, multicentre trial comparing white light bronchoscopy (WLB) with or without AFB. The high-risk group defined by chronic obstructive pulmonary disease plus occupational exposure failed to demonstrate severe dysplasia or carcinoma-in-situ (CIS), although it was unclear to what extent metaplasia or mild dysplasia were seen in this cohort. Swensen et $a l^{4}$ and Bechtel et $a l^{5}$ in two separate studies used bimodality testing using CSC as one portion of their testing and suggested a more significant contribution for CSC in lung cancer detection than was suggested by Loewen et al. ${ }^{2}$ However, because of different study designs including inclusion criteria, biopsy and statistical methods and pathology review variations, ${ }^{6}$ it may be nearly impossible to compare findings from one study to another.

Although Loewen et al raise several compelling clinical questions in their paper, the most pivotal may well be management issues of airway cellular transformation including dysplasia and CIS. The diagnosis, progression and treatment of dysplasia and CIS, especially in high-risk populations, are demanding more clinical attention to determine surveillance strategies and may affect overall outcomes of lung cancer in the near future. Intense interest in this topic was indicated when most sessions at the $11^{\text {th }}$ World Congress on Lung Cancer in 
Barcelona (IASLC) included several abstracts directly or indirectly discussing research or clinical aspects of airway cellular transformation. These presentations ranged from the basic science of biomarkers for cellular transformation to endoscopic detection and surveillance. In clinical thoracic oncology, a discussion of airway transformation usually revolves around three basic questions:

1. What is the natural history of mucosal transformations in the airway?

2. How much cellular transformation needs to be present to warrant treatment: moderate or severe dysplasia, CIS?

3. What is the best approach for detection: routine screening, high risk surveillance or early diagnosis strategies?

Current attempts to address each of these questions are mostly in the form of expert opinion, as seen in a recent review of CIS treatments published by Mathur et al. ${ }^{7}$

Transformation within the central airways remains a pathological diagnosis with obvious grey areas overlapping dysplasia, CIS and frank early carcinoma and, as mentioned earlier, interobserver variability between pathologists on the same specimens is not uncommon. Clinically, there are two important preneoplastic non-small cell presentations within the lung: central airway CIS as a pre-neoplasm of squamous cell carcinoma and peripheral lung atypical adenomatous hyperplasia (AAH) as a pre-neoplasm of bronchoalveolar cell carcinoma and adenocarcinoma. By definition, CIS is radiographically occult, has a surface area of less than $2 \mathrm{~cm}$ with visible margins, and has no invasion beyond the bronchial cartilage. ${ }^{8}$ The prevalence of CIS might be as high as $20-30 \%$ based on the epidemiology of non-small cell lung cancer, but clinical screening or surveillance studies have not been done on the general population and the declining use of autopsies has further limited data collection to support this claim.

The issue of whether dysplasia or CIS is a pre-malignant state or an extreme cellular reaction to injury was explored by Fontanini et $a l^{9}$ who assessed the microvessel count and suggested that, based on this count, a pre-malignant state is not reached until CIS develops. ${ }^{9}$ If it is a pre-malignant state, then the inability to accurately and completely describe the natural history of CIS undermines the ability to truly assess protocols for CIS screening, surveillance and treatment. However, several groups have recently reported data suggesting that pre-malignant cellular transformation does progress to invasive carcinoma, although the relative numbers that progress to frank disease are small and variable. ${ }^{10-12}$ George et $a l^{13}$ have recently reported their experience with a high-risk cohort followed prospectively for several years. They report a cumulative risk of developing lung cancer from high grade lesions in their cohort of $33 \%$ and $54 \%$ at 1 and 2 years.

Pasic et al ${ }^{14}$ defined "early stage" lung cancer as stage I (T1N0, T2NO) and stage II (TlNl, T2Nl) and, as such, dysplasia and CIS (TONOMO or stage 0) do not fall within this definition. Other major epithelial-based tumours such as gastrointestinal and breast cancers rely on screening or surveillance methodology to identify their stage 0 cases and to optimise patient outcomes. CT scanning, positron emission tomography (PET), sputum analysis and bronchoscopy are all used to diagnose and stage early lung cancer, although PET and CT scanning have no established role as a sole modality in the identification of stage $0 .^{15}$ Since CIS is radiographically occult, diagnosis must come from screening populations at risk for neoplastic transformation, performing surveillance on high-risk populations or early diagnosis in patients with suspicious clinical presentations.

Detecting radiographically occult disease relies in part on direct visualisation of the abnormal bronchial mucosa, but the ability to use direct visualisation optimally also depends on why and where CIS develops. Insight into growth patterns and how CIS may progress are important parameters for visualisation strategies. However, despite the ability to assess the entire central airway by standard bronchoscopy and understand its pattern of appearance or association with frank cancer, CIS was infrequently discovered. This has changed with more sophisticated ways to optically analyse airway mucosa, the most significant of which has been AFB. ${ }^{16}$ AF with whitelight bronchoscopy (WLB) has improved both the identification and the extent of airway lesions. ${ }^{13} \quad$ 16-20

AFB has repeatedly shown increased sensitivity when used in combination with WLB over WLB alone. However, the addition of endobronchial ultrasound (EBUS) might improve the specificity of AFB by distinguishing between inflammation and neoplastic penetration of the mucosa. Miyazu et $a^{21}$ demonstrated the ability of EBUS to accurately assess tumour depth.

In addition to optical technologies, diagnosis of airway CIS will include the molecular genetic changes of the progression from metaplasia to invasive carcinoma and the protein products produced by those changes. Detection of that critical point of irreversible transformation to cancer will inevitably include biomarkers and necessitate multimodality strategies.

The paper by Loewen et $a l^{2}$ suffers as a stand alone protocol, limiting cohort size and not allowing comparisons to the other clinical trials looking at surveillance in high-risk populations for lung cancer. It does energise this topic by supporting multimodality approaches and defining a population that may benefit more readily from surveillance strategies. It is obvious that thoracic oncology providers have a forest of more questions than answers for managing patients at risk for lung cancer and, although these issues are daunting, Loewen et al have provided us with one potential path through the woods.

Thorax 2007;62:285-287.

doi: $10.1136 /$ thx.2006.073577

Correspondence to: Dr Gordon H Downie, Brody School of Medicine at East Carolina University, 3E-149 Brody Bld, Greenville, North Carolina 27858, USA

Competing interests: None.

\section{REFERENCES}

1 Henchke Cl, Yankelevitz DF, Libby DM, et al. Survival of patients with stage 1 lung cancer detected on CT screening. N Engl J Med 2006:355:1763-71.

2 Loewen G, Natarajan N, Tan D, et al. Autofluorescence bronchoscopy for lung cancer surveillance based on risk assessment. Thorax 2007;62:335-40

3 Haubinger $\mathrm{K}$, Becker $\mathrm{H}$, Stanzel $\mathrm{F}$, et al. Autofluorescence bronchoscopy with white light bronchoscopy compared with white light bronchoscopy alone for the detection of precancerous lesions. Thorax 2005;60:496-503.

4 Swensen SJ, Jett JR, Midthun DE, et al. Computed tomographic screening for lung cancer. Mayo Clin Proc 2003;78:1 187-8.

5 Bechtel JJ, Kelley WA, Coons TA, et al. Lung cancer detection in patients with airflow obstruction identified in a primary care outpatient practice. Chest 2005; 127:1140-5.

6 Nicholson AG, Perra L, Cury PM, et al. Reproducibility of the WHO/IASLC grading system for pre-invasive squamous lesions of the bronchus. Histopathology $2001 ; 8: 202-8$.

7 Mathur PN, Edell E, Sutedja T, et al. American College of 'Chest Physicians. Treatment of early stage non-small cell lung cancer. Chest 2003;123(1 Suppl): 176-80S.

8 Brever RH, Pasic A, Smit EF, et al. The natural course of preneoplastic lesions in bronchial epithelium. Clin Cancer Res 2005;1 1:537-43.

9 Fontanini G, Vignati S, Bigini D, et al. Neoangiogenesis: a putative marker of malignancy in non-small-cell lung cancer (NSCLC) development. Int J Cancer 1996;67:615-9.

10 Bota S, Auliac JB, Paris C, et al. Follow-up of bronchial precancerous lesions and carcinoma in situ using fluorescence endoscopy. Am J Respir Crit Care Med 2001; 164:1688-93.

11 Miller Y, Keith R, Hirch F, et al. Natural history of untreated endobronchial $\mathrm{CIS}$. Presented at the 11 th ISLAC Meeting, Barcelona: 2005, Abstract P-265.

12 Brever RH, Pasic A, Smit EF, et al. The natural course of pre-neoplastic lesions in bronchial epithelium. Clin Cancer Res 2005; 1 1:537-43.

13 George JM, Banerjee AK, Read CA, et al. Surveillance for the detection of early lung cancer in 
patients with bronchial dysplasia. Thorax 2007;62:43-50

14 Pasic A, Postmus PE, Sutedia TG. What is early lung cancer? A review of the literature. Lung Cancer 2004;45:267-77.

15 Kayani I, Groves AM, Ell PJ, et al. Imaging bronchial carcinoma in situ: possible roles for combined positron emission tomography (PET)-CT. Lancet Oncol 2005;6:190.

16 Lam S, MacAulay C, Hung J, et al. Detection of dysplasia and carcinoma in situ with a lung imaging fluorescence endoscope device. J Thorac Cardiovasc Surg 1993;105:1035-40.

17 Gilbert S, Luketich JD, Christie NA. Fluorescent bronchoscopy. Thorac Surg Clin 2004; 14:71-7

18 Herth FJ, Ernst A, Becker HD. Autofluorescence bronchoscopy: a comparison of two systems (LIFE and D-light) [see comment]. Respiration 2003:70:395-8.

19 Hirsch FR, Prindiville SA, Miller YE, et al. Fluorescence versus white-light bronchoscopy for detection of pre-neoplastic lesions: a

\section{Severity assessment in community- acquired pneumonia: moving on}

\author{
Wei Shen Lim
}

\section{The CURB65 score displays moderate to good discriminatory value in validation studies involving over 11000 patients}

S everity assessment is recognised as a pivotal step in the management of community-acquired pneumonia (CAP). Consequently, much effort over the last three decades has gone into developing tools to aid this process. The Pneumonia Severity Index (PSI) was introduced in 1997 following a study in over 50000 patients and is well established as a robust severity assessment tool in patients with CAP. ${ }^{1}$ The CURB65 and CRB65 scores-which take account of the presence of Confusion, raised Urea (in the case of CURB65), raised Respiratory rate, low Blood pressure and age $>65$ yearswere introduced more recently in $2003 .{ }^{2}$ One of the main benefits of the CURB65 and CRB65 scores is their simplicity in comparison with the PSI which comprises 20 variables. A number of studies over the last 2 years have therefore sought to confirm the value of these scores in different healthcare settings.

In this issue of Thorax, Man et $\mathrm{al}^{3}$ report a large and well conducted validation study of these three severity assessment tools-the PSI, CURB65 and CRB65 scores (see page 348). They recruited 1016 adults with CAP seen in the emergency department of a teaching hospital in Hong Kong and found that all three severity assessment tools performed equally well at discriminating patients into mortality risk groups. The area under the receiver operating characteristic curve (AUC) is a measure of the ability of a test to correctly classify those with and without the outcome in question, and is widely used to describe the performance of these severity assessment tools. The AUC for the PSI, CURB65 and CRB65 scores were $0.74,0.73$ and 0.69 , respectively (a perfect test would have an AUC of 1).

This report raises the current total number of patients studied with respect to the performance of the CURB65 score to over 11000 patients from nine countries: Australia, England, Hong Kong, New Zealand, Scotland, Spain, Sweden, the Netherlands and the United States. ${ }^{24}$ 9 The AUC for the CURB65 score across these validation studies has ranged from 0.73 to 0.87 - that is, moderate to good discriminatory value. In comparing the performance of the PSI and CURB65 score, one study from the US found a small but significant difference in favour of the PSI (AUC 0.76 vs 0.81). ${ }^{6}$ Otherwise, all the other comparative validation studies, including that by Man et al, ${ }^{3}$ have found no significant difference between these two severity assessment tools.

The performance of the CRB65 score has now been studied in over 5000 patients from seven countries. It appears to be comparable to the CURB65 score with AUC values of $0.69-0.86$. The CRB65 score does not require results from any laboratory investigation and is therefore suited to use in the community. However, except for one study from Germany which recruited patients from outpatient clinics, ${ }^{10}$ most of the work with the CRB65 score has been done either in hospitalised patients or in patients initially seen in emergency departments. Further validation of this score in the randomized study. J Natl Cancer Inst 2001;93:1385-91.

20 Ikeda N, Hiyoshi T, Kakihana M, et al. Histopathological evaluation of fluorescence bronchoscopy using resected lungs in cases of lung cancer. Lung Cancer 2003;41:303-9.

21 Miyazu Y, Miyazawa T, Kurimoto N, et al. Endobronchial ultrasonography in the assessment of centrally located early-stage lung cancer before photodynamic therapy. Am J Respir Crit Care Med 2002; 165:832-7. primary care or community setting, where it has greatest applicability, is therefore warranted.

Some studies have tested the PSI and CURB65 score against outcome measures such as the need for ICU admission ${ }^{9}$ or the combined outcome of mortality and/ or need for mechanical ventilation and/or septic shock. ${ }^{11}$ In these situations they perform less well. This is partly because the PSI and CURB65 scores were developed specifically to predict mortality, and also because these other outcome measures are influenced by centre-specific criteria for ICU admission and/or mechanical ventilation. This is reflected in the varying ICU admission rates in different healthcare settings; for instance, the ICU admission rate in the cohort studied by Man $e t$ al $^{3}$ in Hong Kong was $4 \%$ compared with $17 \%$ in a study conducted in Spain. ${ }^{12}$ Importantly, all the validation studies performed in the last few years show that no severity assessment tool, whatever the outcome measure, is perfect (ie, has an AUC of 1), underlining the requirement always to exercise clinical judgement when applying these tools to individual patients.

In last month's Thorax, Barlow et $a l^{7}$ reported a validation study in 419 patients with clinically diagnosed CAP which compared the CURB65 and CRB65 scores with two generic severity assessment tools-the systemic inflammatory response syndrome (SIRS) score and the standardised early warning score (SEWS). They found that the CURB65 and CRB65 scores performed better than the two generic scores (AUC 0.78 for CURB65, 0.73 for CRB65, 0.68 for SIRS and 0.64 for SEWS).

The value of disease-specific severity scores compared with generic severity scores has been a subject of some debate, particularly in the US where severity adjustment scores have been used alongside managed care. The premise underlying generic scores is that illness severity is a universal concept based on derangements in physiology. Therefore, generic scores allow comparison of patients across different diseases. Conversely, disease-specific scores are based on the 